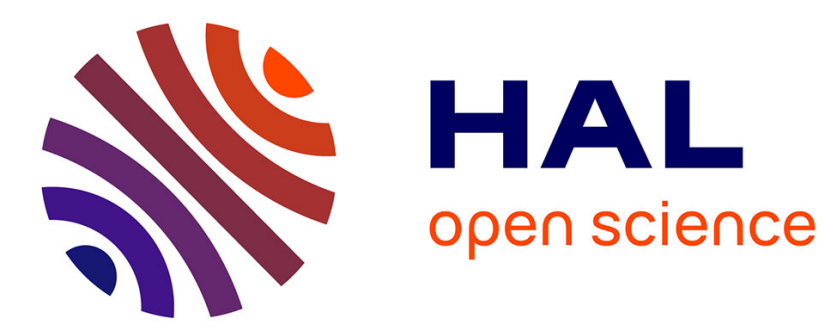

\title{
An industrial security system for human-robot coexistence
}

Philip Long, Christine Chevallereau, Damien Chablat, Alexis Girin

\section{To cite this version:}

Philip Long, Christine Chevallereau, Damien Chablat, Alexis Girin. An industrial security system for human-robot coexistence. Industrial Robot: An International Journal, 2018, 45 (2), pp.220-226. 10.1108/IR-09-2017-0165 . hal-01671012

\section{HAL Id: hal-01671012 https://hal.science/hal-01671012}

Submitted on 27 Dec 2017

HAL is a multi-disciplinary open access archive for the deposit and dissemination of scientific research documents, whether they are published or not. The documents may come from teaching and research institutions in France or abroad, or from public or private research centers.
L'archive ouverte pluridisciplinaire HAL, est destinée au dépôt et à la diffusion de documents scientifiques de niveau recherche, publiés ou non, émanant des établissements d'enseignement et de recherche français ou étrangers, des laboratoires publics ou privés. 


\section{An Industrial Security System for Human-Robot Coexistence Philip Long \\ Department of Electrical and Computer Engineering, Northeastern University, Boston, Massachusetts, USA \\ Christine Chevallereau and Damien Chablat \\ Laboratoire des sciences du numérique de Nantes, Centre National de la Recherche Scientifique, Nantes, France Alexis Girin \\ Department of Robotics and Cobotics, Institut de Recherche Technologique Jules Verne, Bouguenais, France}

Purpose - The installation of industrial robots requires security barriers, a costly, time consuming exercise. Collaborative robots may offer a solution, however these systems only comply with safety standards if operating at reduced speeds. This paper describes the development and implementation of a novel security system that allows human robot co-existence while permitting the robot to execute much of its task at nominal speed.

Design/methodology/approach - The security system is defined by three modes: a nominal mode, a coexistence mode and a gravity compensation mode. Mode transition is triggered by three lasers, two of which are mechanically linked to the robot. These scanners create a dynamic envelope around the robot and allow the detection of operator presence or environmental changes. To avoid velocity discontinuities between transitions we propose a novel time scaling method. Findings - The paper describes the system's mechanical, software and control architecture. The system is demonstrated experimentally on a collaborative robot and is compared with the performance of a state of art security system. Both a qualitative and quantitative analysis of the new system is carried out.

Pratical Implications - The mode transition method is easily implemented, requires little computing power and leaves the trajectories unchanged. As velocity discontinuities are avoided, motor wear is reduced. The execution time is substantially less than a commercial alternative. These advantages can lead to economic benefits in high volume manufacturing environments.

Originality/value - This paper proposes a novel system that is based on industrial material but that can generate dynamic safety zones for a collaborative robot.

Keywords: Collaborative Robots (Cobots); Safety; Flexible manufacturing; Human-Robot Interaction; Time Scaling.

Paper type: Research paper

\section{Introduction}

The integration of industrial robots in manufacturing can increase both efficiency and precision while reducing overall costs. However, the dangers associated with robots limit the potential benefits of operator/robot interaction. Moreover, despite stringent security procedures, accidents do occur [Vasic and Billard, 2013, Jiang and Gainer, 1987, Murashov et al., 2016]. Collaborative robots are designed to interact with humans thanks to their lightweight structure, flexible links and compliant actuators [Bischoff et al., 2010]. Provided defined power and speed limits are respected [ISO15066, 2016, Kock et al., 2006], these systems do not require protective structures, unlike their industrial counterparts [ISO10218, 2011]. This leads to a reduced factory footprint which in turn means that the installation area is no longer off-limits to operators. Finally, advanced collaborative robots can be used to physically assist operators with difficult tasks thus improving task ergonomics and decreasing cycle time [Helms et al., 2002], [H“agele et al., 2002] , [Cherubini et al., 2016].

Robotic security schemes can be broadly defined as post-collision or pre-collision [Heinzmann and Zelinsky, 2003] systems. Post-collision systems react after collision and are typically not equipped with exteroceptive sensing abilities. Safety is assured by limiting the maximum transmittable energy during impact [Haddadin et al., 2009], due to the robot's compliant structure or by using the measured force in the control scheme [Salisbury, 1980, Hogan, 1985]. Passivity control strategies can be used to ensure saftey when physical human robot interaction is desired, for instance in haptic enabled rehabilitation tasks [Atashzar et al., 
2017]. Post-collision schemes are by definition reactive and hence suffer from two significant drawbacks. Firstly, a collision could be dangerous if limits are poorly defined, especially if the robot is carrying a sharp object or tool. Secondly, any collision will halt task execution leading to a decrease in production line efficiency.

In contrast, pre-collision schemes exploit exteroceptive sensors to detect human or obstacle presence. This allows the robot to stop or alter its trajectory before collision occurs. Industrial examples include the Bosch APAS system and ABB SafeMove [Behnisch, 2008], where proximity sensors detect operator presence and prevent a potentially dangerous collision. These systems create large static zones around the robot, where any intrusion decreases the task execution rate. Thus recent work has focused on reducing the size of this zone and modifying robot behavior only if there is an imminent danger of collision. In [Rybski et al., 2012], 3D imaging sensors are used to create a volumetric representation of the zones occupied by operator and by the robot. If these zones overlap, the robot slows down or stops. Likewise in [Zhang et al., 2016], a 3D camera is used to detect a operator after which potential field method is used to generate a collision free path. In [Mohammed et al., 2017,Schmidt and Wang, 2014] a virtual 3D robot model is used with depth images of human operators to pre-empt collisions.

The operator's 3D position is also tracked in [Kulić and Croft, 2006, Bascetta et al., 2011], where the current position and the operator's intended position are used to alter the robot's behavior. The idea of calculating operator risk is explored in [Ikuta et al., 2003], where the authors showed that any danger index should be based on relative position, velocity [Tsai et al., 2014] and posture. Likewise in [Kulić and Croft, 2006, Kulić and Croft, 2007], the relative position, velocity and effective inertia is calculated at the point of minimum distance between human and robot. Relative position and velocity is also used as a safety metric in an industrial setting in [Zanchettin et al., 2016], while in [Lasota et al., 2014], the robot's velocity is decreased in response to an operator's approach. Finally, an interesting approach is proposed in [Lacevic and Rocco, 2010] and [Ragaglia et al., 2014] where a danger field based uniquely on the robot's state is generated. Thus an intended intrusion into this zone can be used to modify robot behavior.

While the above methods are very promising, they lack a maturity in the industrial setting. In this paper we propose a security scheme tailored to the industrial environment. Thus in contrast to previous works, the security aspects of this system are based on the use of a certified collaborative robot that ensures human safety when reduced velocity modes are activated. We propose to add on-board laser sensors that create a spatial envelope around the robot which, in the case of a positive detection, trigger a reduced velocity mode. The objectives of this work are twofold. Firstly to ensure the system allows human robot coexistence i.e. eliminating the need of protective structures. Secondly to ensure that task efficiency is not sacrificed for safety by minimizing the time spent in low speed modes. In addition to this we propose a novel and simple approach to change robots operating speeds without inducing acceleration discontinuities. 


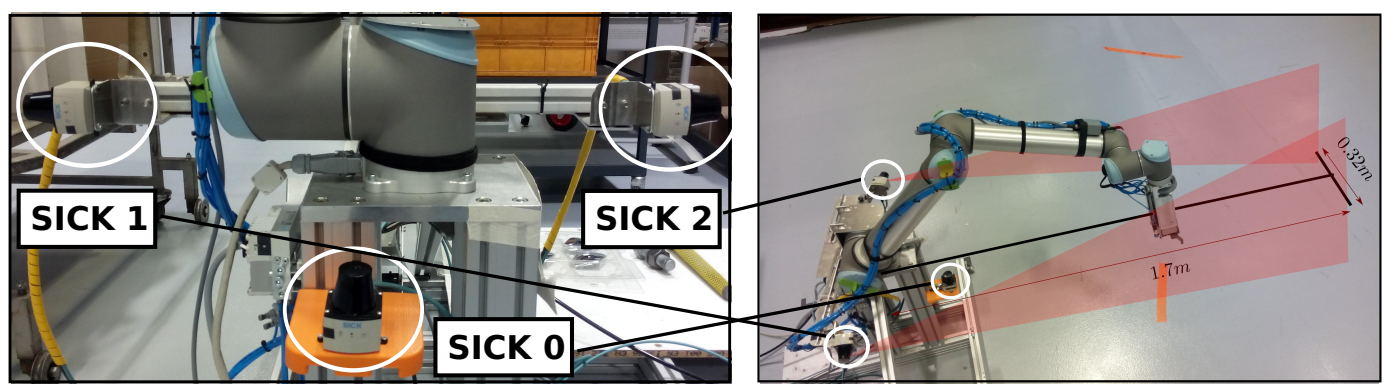

Fig. 1: Mechanical architecture of security system creating dynamic detection zone.

\section{System Description}

The system comprises Universal Robot's UR10, a collaborative robot with a certified postcollision safety system, and three exteroceptive sensors. The robot's task can be achieved by executing a set of trajectories. During the task, the operator may work in close proximity to the robot, for instance collecting a prepared kit, and may freely enter and exit the workspace at unknown locations/times. Task cooperation is not treated in this paper nevertheless the robot's actions must not pose a risk to the operator's safety. The task must be completed in the minimum time without restarting the robot or triggering a security stop. It should be noted that the static environment is learned off-line (by executing a pre-defined motion and recording sensor output) and filtered from the detection point cloud.

The robot can operate in different security modes which can be selected by an external digital input. In Nominal mode, the robot operates at its maximum velocity. In Reduced mode, the robot operates at diminished capacity with limits on velocity and power that respect human robot collaboration norms defined in [ISO15066, 2016]. Finally, in Passive mode the robot compensates its own weight, often known as gravity compensation mode.

Our security scheme is based on three SICK TiM551 lasers, each of which creates a detection plane. Two lasers are mechanically linked to robot's first axis creating two vertical planes. These planes move with the robot to define a dynamic detection zone. To ensure that the operator is detected at a reasonable distance, these planes are inclined rather than parallel. The third laser creates a static horizontal plane positioned such that the operator's legs are detected upon zone entry. The mechanical architecture is shown in Fig. 1.

\subsection{Security System Description}

The main idea is to create a 3D dynamic zone that envelopes the robot. An operator will be detected if he/she intersects any of the three laser planes shown in Fig. 1. A detection will modify the security modes if the distance is small enough to constitute a danger.

Suppose the sensors have detected several objects in the robot's workspace. In the laser plane each object is described by polar coordinates. The $k^{\text {th }}$ object's polar position is denoted $\left(r_{k}, \theta_{k}\right)$. The object's homogeneous position with respect to sensor $s$ is given as ${ }^{s} \mathbf{p}_{k}=$ 


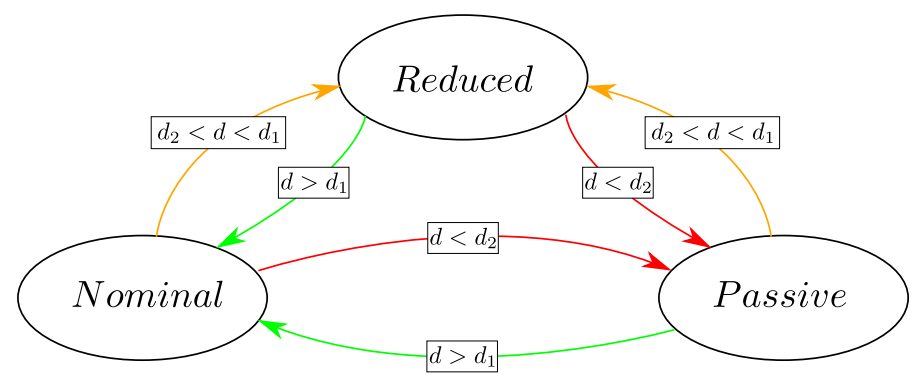

Fig. 2: Security state machine: $d$ is the object distance and $d_{1} d_{2}$ represent the thresholds

$\left[r_{k} \cos \left(\theta_{k}\right), r_{k} \sin \left(\theta_{k}\right), 0,1\right]^{T}$. The position in the fixed frame is calculated as

$$
{ }^{w} \mathbf{p}_{k}={ }^{w} \mathbf{T}_{s}{ }^{s} \mathbf{p}_{k} .
$$

where ${ }^{w} \mathbf{T}_{s}$ is the known transformation matrix from the sensor to the world frame [Khalil and Dombre, 2002]. The distance between the object and the robot's $n$ link frames is calculated, and the minimum distance is retained,

$$
d_{k}=\min \left(\left\|{ }^{w} \mathbf{p}_{k}-{ }^{w} \mathbf{p}_{1}\right\|,\left\|{ }^{w} \mathbf{p}_{k}-{ }^{w} \mathbf{p}_{2}\right\| \ldots\left\|^{w} \mathbf{p}_{k}-{ }^{w} \mathbf{p}_{n}\right\|\right),
$$

where $\left\|{ }^{w} \mathbf{p}_{k}-{ }^{w} \mathbf{p}_{i}\right\|$ is the distance between the $k^{t h}$ point and the $i^{\text {th }}$ frame. ${ }^{\text {a }}$ Finally $d$ is obtained as the minimum distance between all detected objects and the robot i.e.,

$$
d=\min \left(d_{1}, d_{2}, \ldots d_{k}, \ldots d_{n},\right) .
$$

$d$ is used to trigger the three security modes. A conceptual view of the system is given in Fig. 2 while Fig. 3 shows the zones with respect to the robot.

\section{Security Mode change via time scaling}

The proposed mode change can be implemented on the UR10 using configurable digital inputs of $24 \mathrm{~V}$. However, this method leads to a discontinuity in the desired velocity which in turn generates structural vibrations that may damage the robot. Moreover, these discontinuities can generate inertial body forces that are sufficiently large to trigger a security stop. In this paper, we propose time scaling as a means of reducing discontinuities when transitioning between operational modes.

The robot's trajectory, $\mathcal{Q}$, is composed of a series of positions with associated velocities and times, denoted for $i=1 \ldots n, \mathbf{q}_{i}, \dot{\mathbf{q}}_{i}$ and $t_{i}$ respectively. $\mathcal{Q}$ is given as

$$
\mathcal{Q}=\left[\begin{array}{ccc}
t_{1} & \mathbf{q}_{1} & \dot{\mathbf{q}}_{1} \\
t_{2} & \mathbf{q}_{2} & \dot{\mathbf{q}}_{2} \\
\vdots & \vdots & \vdots \\
t_{n} & \mathbf{q}_{n} & \dot{\mathbf{q}}_{n}
\end{array}\right]
$$

${ }^{\text {a }}$ For the horizontal laser only the $x-y$ coordinates are considered in this calculation. 

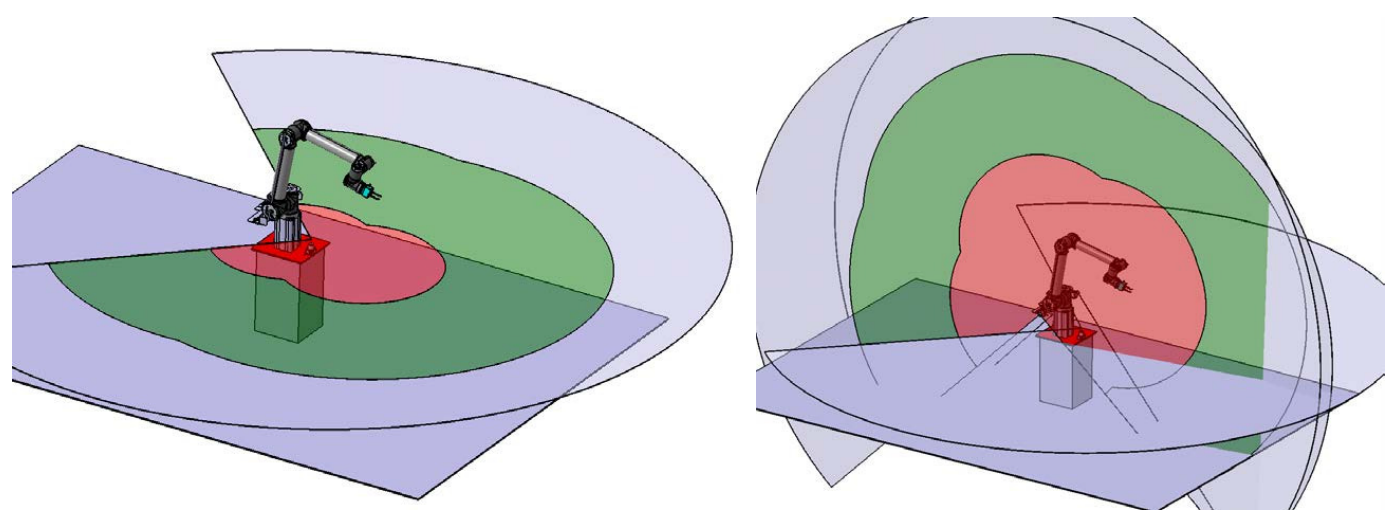

Fig. 3: System's security zones. Green and red show the zones where a detection generates a reduced and passive request respectively. (Left) Zones for the horizontal laser. (Right) Zones for vertical lasers. It should be noted that while the horizontal laser is fixed, the security zones in this plane are dynamic since they evolve with the robot's motion.

At every $\Delta t$ seconds (the communication rate) a cubic interpolation calculates the desired joint position. For example, at time $t_{i}+\Delta t$, the desired position is obtained as

$$
\mathbf{q}^{d}=\operatorname{interpolate}\left(\mathcal{Q}, t_{i}+\Delta t\right) .
$$

The robot moves from, $\mathbf{q}_{i}$, to $\mathbf{q}_{d}$ in $\Delta t$, resulting in a velocity of

$$
\dot{\mathbf{q}}=\frac{\mathbf{q}^{d}-\mathbf{q}_{i}}{\Delta t} .
$$

In our proposed method, the time used for the interpolation is varied by a rate reduction factor $k$, where $0<k<1$. Thus the desired position becomes

$$
\mathbf{q}_{v t}^{d}=\operatorname{interpolate}\left(\mathcal{Q}, t_{i}+k \Delta t\right),
$$

where, since the trajectory is continuous in position and $k<1,\left\|\mathbf{q}_{v t}^{d}-\mathbf{q}_{i}\right\|<\left\|\mathbf{q}^{d}-\mathbf{q}_{i}\right\|$. To reach the desired configuration the robot still has $\Delta t$ seconds, as the rate of communication remains unchanged, resulting in a velocity of

$$
\dot{\mathbf{q}}_{v t}=\frac{\mathbf{q}_{v t}^{d}-\mathbf{q}_{i}}{\Delta t} .
$$

Hence, it can be seen that

$$
\dot{\mathbf{q}}_{v t}=k \dot{\mathbf{q}} \quad \text { while } \quad \ddot{\mathbf{q}}_{v t}=k^{2} \ddot{\mathbf{q}} .
$$

Thus the same trajectory $\mathcal{Q}$ is followed in position while the resulting velocity and acceleration are reduced. A comparison between mode changes with and without this method is shown in Fig 4. 


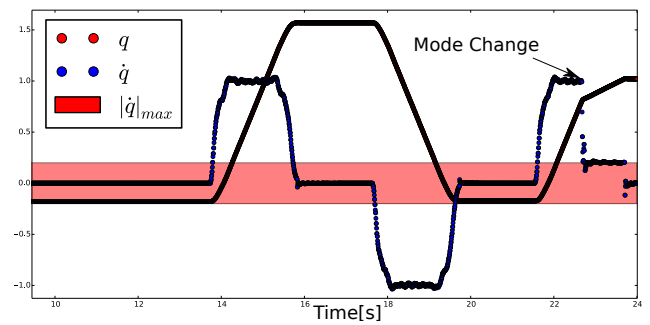

(a)

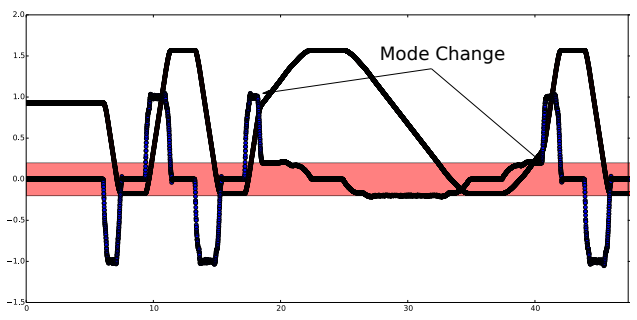

(b)

Fig. 4: Comparison of mode change by using (a) internal robot controller and (b) with time scaling.

\section{Experimental validation}

The management of the security modes, integration of the sensor data and trajectory generation are executed on an industrial PC which sends a desired joint position to the robot every $2 \mathrm{~ms}$. For these tests rate reduction factor is given as $k=0.2$ i.e. one fifth of the maximum velocity.

\subsection{Qualitative validation}

Five operators repeated six scenarios five times (a total of 150 tests). Each scenario required the operator to enter and exit by a defined corridor while the robot is in a particular zone. The six scenarios are shown in Fig.5. Of the five operators, three had no knowledge of the security system in place. After each sequence the operator responded to a questionnaire, from which the following observations have been made:

(1) Throughout the tests, the system conformed to expected behavior; no security stop
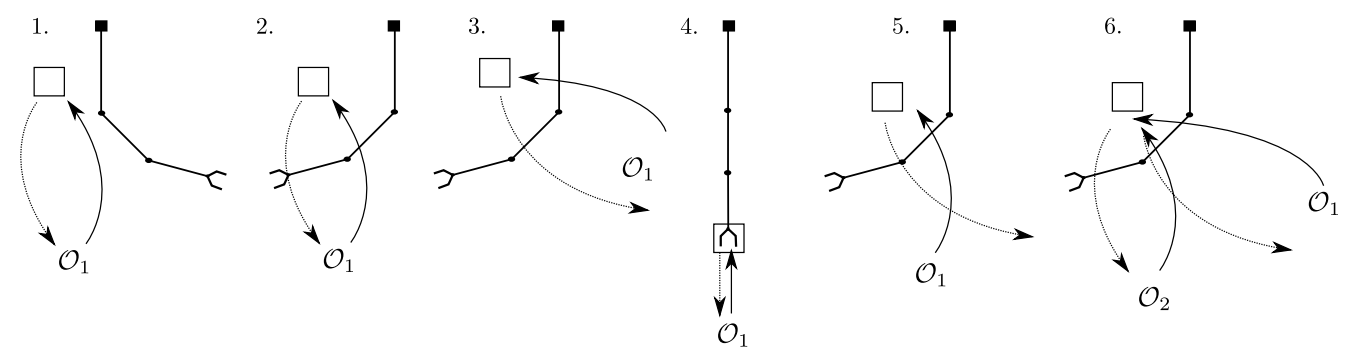

Fig. 5: Scenario 1-6 for qualitative evaluation of security. The robot executes a cyclic motion task between workspace extremities. During the task, the operator moves from the initial position at $\mathcal{O}_{1}$ to the white square where she/he executes a simple action and then leaves by the second arrow. The robot's configuration is shown at the moment where the operator enters in the workspace. For scenario (6), two operators work together. 
occurred and the robot changed its mode according operator proximity. The operator reported no feelings of anxiousness or stress as the behavior of the robot was seen as predictable.

(2) The operators understood the passive mode and felt at ease displacing the robot. This contrasts to a previously employed reactive controller, whereby if the operator-robot distance, obtained from local sensors, is less than a predefined threshold the robot alters its trajectory. Once the distance is greater than the threshold the robot reverts to its original trajectory. This autonomous motion towards the operator was deemed somewhat distressing.

(3) When exiting the passive mode the robot's trajectory is re-planned since the operator may have displaced it by hand. Excluding this case, the robot's desired trajectory is unmodified and for a repetitive task quickly becomes familiar for the operator. Previous employed security schemes focused on on-line trajectory definition to avoid operators or objects. However, workspace limitations coupled with the desire to reduce planning time led to unanticipated motions (especially when the robot reconfigured to avoid joint limits). This behavior stresses the operator.

(4) The smooth transition of mode without vibration, due to the time scaling system, also increases the system's acceptability for the operator.

An example video for one operator is available at: https://youtu.be/dhBb18IlNAO

\subsection{Quantitative validation}

For three different tasks, consisting of several operator entries and exits, sensor and temporal data was recorded to permit a quantitative validation. In this case the limits for reduced and passive mode were set as 1 meter and 0.5 meters respectively ${ }^{\mathrm{b}}$. Table 1 shows the system's performance, with respect to the optimal performance, including the extra task time required to ensure operator safety. Fig. 6 shows which sensors are responsible for generating the security request. The vertical sensors are particularly important for initial detection in the passive zone, as they can detect outstretched arms, whereas the reduced mode is most often triggered by the horizontal laser i.e. a leg detection.

\subsection{Comparison with industrial system}

\subsubsection{System Description}

Our security scheme, $A$, is compared with a generic system, $B$, representing the state of the art. Scheme $B$ consists of one fixed horizontal laser, placed below the robot base, as shown in Fig 8, whereas $A$, as described in Section 2, consist of this laser in addition to two sensors mechanically attached to the robot. Two security zones are defined for scheme $B$ but these zones are static as proposed by commercial systems.

\footnotetext{
b In reality, these limits should be fixed with respect to the robot's braking distance, human walking speed,
} tool type and task type. 


\begin{tabular}{|c|c|cc|c|c|c|}
\hline \hline \multirow{2}{*}{$\begin{array}{c}\text { Test } \\
\text { no. }\end{array}$} & Theoretical & \multicolumn{4}{|c|}{ Actual Active Time } & \\
& Duration & Nominal & Reduced & Compensation & Time & Time \\
lost
\end{tabular}

Table 1: Theoretical Active Time is the total theoretical duration of all executed trajectories. Actual Trajectory Time is the actual duration of the task decomposed into the time spent in each security mode. The time lost is simply the difference between optimum task execution time and actual task execution time.

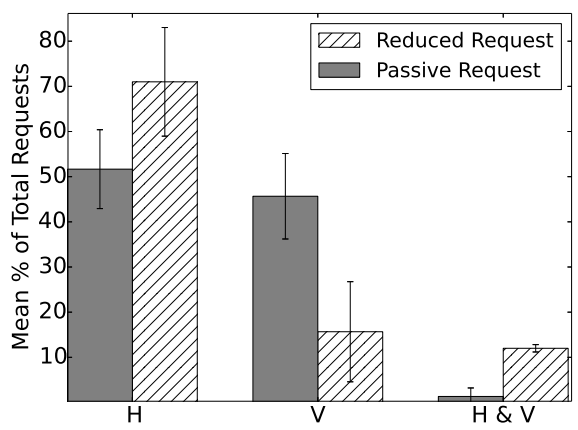

\begin{tabular}{|c|ccc|ccc|}
\hline Test & \multicolumn{3}{|c|}{ Reduced } & \multicolumn{3}{c|}{ Passive } \\
no. & \multicolumn{3}{|c|}{ Request } & \multicolumn{3}{c|}{ Request } \\
& $\mathrm{H}$ & $\mathrm{V}$ & $\mathrm{H} \& \mathrm{~V}$ & $\mathrm{H}$ & $\mathrm{V}$ & $\mathrm{H} \& \mathrm{~V}$ \\
\hline 1 & 27 & 10 & 6 & 9 & 13 & 0 \\
2 & 32 & 0 & 4 & 12 & 9 & 1 \\
3 & 21 & 8 & 4 & 8 & 5 & 0 \\
\hline \hline
\end{tabular}

Fig. 6: Security requests evoked by sensors. H, V, H\&V denote whether the request is triggered by the horizontal, vertical or both simultaneously, respectively. The chart shows the mean percentage of total detections with standard deviation.

For system A, an operator's limb can be detected by a vertical plane. The distance which triggers a mode change between the robot's axes and an obstacle is set to 1.0 meter for the reduced mode and 0.5 meters for the passive mode. However, in order to maintain the same security level for system B, the UR10's reach (1.3 meters) must be consider since the distance is measured to the sensor frame and the robot configuration is unknown. Furthermore, an outstretched limb (estimated at $0.5 \mathrm{~m}$ ) must also be considered since only the lower body can be detected by the horizontal plane. Hence, for the same security level the zone limits for $B$ are greater than $A$. B's reduced and passive limits are defined as 2.8 and 2.0 meters respectively ${ }^{c}$. It should be noted that the distance calculation differs in the two cases. For system B, the reference (the sensor frame) is fixed, whereas for system A, the distance is measured to the robot's axes.

${ }^{\mathrm{c}}$ The difference in offsets between passive and reduced is due to the use of the vertical planes which are offset from the robot. 


\begin{tabular}{|c|ccc|ccc|}
\hline \hline & \multicolumn{3}{|c|}{$\mathrm{A}$} & \multicolumn{3}{c|}{$\mathrm{B}$} \\
& \% Nominal & \% Reduced & \% Gravity & \% Nominal & \% Reduced & \% Gravity \\
\hline 1 & 52.80 & 11.64 & 35.56 & 22.41 & 25.26 & 52.33 \\
2 & 55.10 & 15.12 & 29.77 & 18.89 & 28.93 & 52.20 \\
3 & 70.48 & 12.42 & 17.15 & 32.46 & 31.00 & 36.53 \\
\hline$\mu$ & 59.5 & 13.1 & 27.5 & 24.59 & 28.4 & 47.02 \\
\hline \hline
\end{tabular}

Table 2: Temporal comparison of A and B for the three experiments, where $\mu$ is the mean value.

\subsubsection{Comparison}

Since both systems assure the operator's safety, the performance is compared with respect to the total time required to execute a given task i.e., those described in Section 4.2. The reduced and passive zones are larger for $B$ than for $A$. Therefore for a given operator presence, the robot will spend more time in a low-speed mode when scheme $B$ is used. It follows that a system equipped security scheme $B$ will take longer to execute the same set of motions.

The time spent in each mode as a percentage of the total active duration is used as a comparative metric, as shown in Table $2^{\mathrm{d}}$ and graphically in Fig. 7. This percentage is evaluated from experiments executed while robot is equipped with system $A$. At each instant, using the robot configuration and the operator's pose, the security mode is calculated for the system $A$ and for a virtual system $B$. For instance the first row of Table 2 (Test 1 ) shows that system $A$ spends $52.80 \%$ of the total test time in nominal mode, $11.64 \%$ in reduced mode and $35.56 \%$ in passive mode. During this test, if the system had been equipped with security scheme $B$ the percentage of time in the reduced and passive modes would increase to $25.26 \%$ and $52.33 \%$, respectively. The increase in time spent in the reduced and passive modes decreases the productivity by augmenting the time required to execute a defined series of motions.

The last row in Table 2 compares the mean percentage of time. Our objective is to quantify the robot's effective mean velocity for the two security systems. For example, suppose the robot follows a path of length $p$ in a time $T$ (i.e., the total time given in Table 1). $p$ can be written as

$$
p=p_{N}+p_{R},
$$

where $p_{N}$ denotes the part of the path executed at nominal velocity $v$ and $p_{R}$, the part at reduced velocity at $k \cdot v$. By definition, the robot is in nominal mode for $\%$ Nominal $T$ seconds, reduced mode for \%Reduced.T seconds and passive mode for \%Gravity.T seconds,

\footnotetext{
${ }^{\mathrm{d}}$ In this study, for a given motion type obtained using our system A, the zone (nominal, reduced, gravity) is calculated based on the current position of the robot and the object considering both the system A and B. An over estimation of the duration of the reduced mode is thus obtained for the system B, since the real velocity of the robot is defined by system $A$.
} 


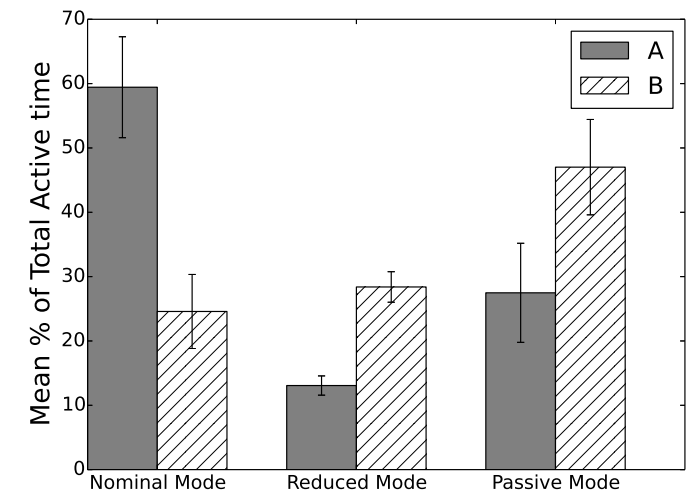

Fig. 7: Time spent in each mode as a percentage of total active time)

therefore

$$
p_{N}=\% \text { Nominal } \cdot T \cdot v, \quad \quad p_{R}=\% \text { Reduced } \cdot T \cdot k \cdot v .
$$

The mean effective velocity is calculated by

$$
v_{\text {eff }}=\frac{p}{T}=\frac{p_{N}+p_{R}}{T}=\% \text { Nominal } \cdot v+\% \text { Reduced } \cdot k \cdot v
$$

By using (12), the effective velocity for the two security systems is given as

$$
\begin{aligned}
& v_{A}=0.595 \cdot v+0.131 \cdot 0.2 \cdot v=0.6212 v \\
& v_{B}=0.2459 \cdot v+0.284 \cdot 0.2 \cdot v=0.3027 v
\end{aligned}
$$

In order to compare the two systems, a performance ratio may be defined as follows

$$
\frac{v_{A}}{v_{B}}=\frac{\% \operatorname{Nominal}_{A}+\left(\% \operatorname{Reduced}_{A} \cdot k\right)}{\% \operatorname{Nominal}_{B}+\left(\% \operatorname{Reduced}_{B} \cdot k\right)}=2.0522 .
$$

Consequently, for a set of tasks with frequent operator presence, our system's effective velocity will be twice as than high as the generic security system, $B$, reducing the task duration by two.

\section{Conclusion}

This paper has described a security system that allows human robot co-existence in an industrial environment. This work has shown how the use of dynamic security zones significantly increases the robot's performance in terms of average velocity with respect to static security zone systems. There are two main contributions in this work. Firstly, an innovative system layout that creates a 3D detection zone around the robot with industrial laser scanners. In contrast to previous works the use of laser scanners is attractive due to their fast response and reliability in an industrial setting. Secondly, we have proposed a method of transitioning between different velocity modes that avoids discontinuities in desired velocity. 
This method is easily implemented, requires very little computing power and leaves the path unchanged. The robot executes the current trajectory during rate changes thus eliminating planning time and unnecessary stops. Our system was shown to perform better than a generic system that uses static zones to detect operator presence, requiring half the time to execute the same task. Moreover, the system performed very robustly over a series of tests and was generally well received by the operators. It necessitates no human intervention to restart the robot after security stops and as velocity discontinuities are avoided, motor wear is reduced. The above advantages can lead to significant economic benefits in high volume manufacturing environments.

The current limitations in this work, include the mechanical constraints applied to the robot by the sensor mechanism and the use, currently, of uncertified security sensors. Future work will focus on implementing this scheme on a security certified industrial setting.

\section{Acknowledgments}

\section{References}

Atashzar, S. F., Shahbazi, M., Tavakoli, M., and Patel, R. V. (2017). A grasp-based passivity signature for haptics-enabled human-robot interaction: Application to design of a new safety mechanism for robotic rehabilitation. The International Journal of Robotics Research, page 0278364916689139 .

Bascetta, L., Ferretti, G., Rocco, P., Ardö, H., Bruyninckx, H., Demeester, E., and Lello, E. D. (2011). Towards safe human-robot interaction in robotic cells: an approach based on visual tracking and intention estimation. In IEEE/RSJ Int. Conf. on Intelligent Robots and Systems, pages 2971-2978. IEEE.

Behnisch, K. (2008). White paper safe collaboration with abb robots electronic position switch and safemove. ABB, Walnut Creek, CA, USA.

Bischoff, R., Kurth, J., Schreiber, G., Koeppe, R., Albu-Schäffer, A., Beyer, A., Eiberger, O., Haddadin, S., Stemmer, A., Grunwald, G., et al. (2010). The kuka-dlr lightweight robot arm-a new reference platform for robotics research and manufacturing. In Int. symposium on Robotics, pages 1-8. VDE.

Cherubini, A., Passama, R., Crosnier, A., Lasnier, A., and Fraisse, P. (2016). Collaborative manufacturing with physical human-robot interaction. Robotics and Computer-Integrated Manufacturing, 40:1-13.

Haddadin, S., Albu-Schäffer, A., Frommberger, M., Rossmann, J., and Hirzinger, G. (2009). The dlr crash report: towards a standard crash-testing protocol for robot safety-part ii: discussions. In IEEE Int. Conf. on Robotics and Automation, pages 280-287. IEEE.

Hägele, M., Schaaf, W., and Helms, E. (2002). Robot assistants at manual workplaces: Effective co-operation and safety aspects. In Int. Symposium on Robotic, pages 7-11.

Heinzmann, J. and Zelinsky, A. (2003). Quantitative safety guarantees for physical human-robot interaction. The International Journal of Robotics Research, 22(7-8):479-504.

Helms, E., Schraft, R. D., and Hägele, M. (2002). Robot assistant in industrial environments. In IEEE Int. Workshop on Robot and Human Interactive Communication, pages 399-404. IEEE.

Hogan, N. (1985). Impedance control: An approach to manipulation: Part ii implementation. Journal of dynamic systems, measurement, and control, 107(1):8-16.

Ikuta, K., Ishii, H., and Nokata, M. (2003). Safety evaluation method of design and control for human-care robots. The International Journal of Robotics Research, 22(5):281-297.

ISO10218 (2011). Robots and robotic devices - safety requirements for industrial robots. Technical report, International Organization for Standardization. 
ISO15066 (2016). Robots and robotic devices - collaborative robots. Technical report, International Organization for Standardization.

Jiang, B. C. and Gainer, C. A. (1987). A cause-and-effect analysis of robot accidents. Journal of Occupational accidents, 9(1):27-45.

Khalil, W. and Dombre, E. (2002). Modeling Identification and Control of Robots. Hermes-Penton, London.

Kock, S., Bredahl, J., Eriksson, P. J., Myhr, M., and Behnisch, K. (2006). Taming the robot: better safety without higher fences. ABB Review, 4:11-14.

Kulić, D. and Croft, E. (2007). Pre-collision safety strategies for human-robot interaction. $A u$ tonomous Robots, 22(2):149-164.

Kulić, D. and Croft, E. A. (2006). Real-time safety for human-robot interaction. Robotics and Autonomous Systems, 54(1):1-12.

Lacevic, B. and Rocco, P. (2010). Kinetostatic danger field-a novel safety assessment for humanrobot interaction. In 2010 IEEE/RSJ Int. Conf. on Intelligent Robots and Systems, pages 21692174. IEEE.

Lasota, P., Rossano, G. F., Shah, J., et al. (2014). Toward safe close-proximity human-robot interaction with standard industrial robots. In IEEE Int. Conf. on Automation Science and Engineering, 2014, pages 339-344. IEEE.

Mohammed, A., Schmidt, B., and Wang, L. (2017). Active collision avoidance for human-robot collaboration driven by vision sensors. International Journal of Computer Integrated Manufacturing, 30(9):970-980.

Murashov, V., Hearl, F., and Howard, J. (2016). Working safely with robot workers: Recommendations for the new workplace. Journal of occupational and environmental hygiene, 13(3):D61-D71.

Ragaglia, M., Bascetta, L., Rocco, P., and Zanchettin, A. M. (2014). Integration of perception, control and injury knowledge for safe human-robot interaction. In IEEE Int. Conf. on Robotics and Automation, pages 1196-1202. IEEE.

Rybski, P., Anderson-Sprecher, P., Huber, D., Niessl, C., and Simmons, R. (2012). Sensor fusion for human safety in industrial workcells. In IEEE/RSJ Int. Conf. on Intelligent Robots and Systems, pages 3612-3619. IEEE.

Salisbury, J. K. (1980). Active stiffness control of a manipulator in cartesian coordinates. In IEEE Conf. on Decision and Control including the Symposium on Adaptive Processes, pages 95-100. IEEE.

Schmidt, B. and Wang, L. (2014). Depth camera based collision avoidance via active robot control. Journal of manufacturing systems, 33(4):711-718.

Tsai, C.-S., Hu, J.-S., and Tomizuka, M. (2014). Ensuring safety in human-robot coexistence environment. In IEEE/RSJ Int. Conf. on Intelligent Robots and Systems, pages 4191-4196. IEEE.

Vasic, M. and Billard, A. (2013). Safety issues in human-robot interactions. In IEEE Int. Conf. on Robotics and Automation, pages 197-204. IEEE.

Zanchettin, A. M., Ceriani, N. M., Rocco, P., Ding, H., and Matthias, B. (2016). Safety in humanrobot collaborative manufacturing environments: Metrics and control. IEEE Transactions on Automation Science and Engineering, 13(2):882-893.

Zhang, P., Jin, P., Du, G., and Liu, X. (2016). Ensuring safety in human-robot coexisting environment based on two-level protection. Industrial Robot: An International Journal, 43(3):264-273. 